\title{
Discotic materials for organic solar cells: Effects of chemical structure on assembly and performance
}

\author{
Holger Christian Hesse ${ }^{a}$, Jonas Weickert ${ }^{a}$, Mahmoud Al-Hussein ${ }^{b}$, Lukas Dössel ${ }^{c}$, Xinliang Feng ${ }^{c}$, \\ Klaus Müllen ${ }^{c}$, Lukas Schmidt-Mende ${ }^{a, *}$ \\ ${ }^{a}$ Ludwig-Maximilians-University Munich, Department of Physics and Center for NanoScience (CeNS), Amalienstr. 54, 80799 Munich, Germany \\ ${ }^{\mathrm{b}}$ Department of Physics, University of Jordan, Amman 11942, Jordan \\ ${ }^{\mathrm{c}}$ Max Planck Institute for Polymer Research, Ackermannweg 10, 55128 Mainz, Germany
}

Keywords:

Organic photovoltaics

Discotic materials

Inverted solar cell

Self-assembly

Transient decay measurements

\begin{abstract}
A B S T R A C T
Discotic materials have attracted remarkable interest for application in organic solar cells. We analyze a series of phenyl substituted hexa peri hexabenzocoronenes (HBCs) with residue modifications blended with perylenediimide (PDI) as donor material. The effect of differing alkyl chain lengths of $6,8,12$ and 16 carbon atoms, introduction of a triple bond linker between HBC core and residual phenyl group and a swallow tailed dialkylphenyl chain on the device performance is investigated. Detailed insight to device physics and morphology is gained by analysis of photoluminescence quenching, transient photovoltage and photocurrent decay experiments and atomic force microscopy. Complementary the investigations explain why using short alkyl side chains higher currents and consequently increased device performance can be achieved. We report an external quantum efficiency of over $27 \%$ for devices based on discotic molecules. In this work the molecular assembly and its impact on performance in photovoltaic devices is studied. The study also reveals pathways to further increase the quantum yield of small molecule organic solar cells.
\end{abstract}

\section{Introduction}

Fabrication of photovoltaic devices using solution processable materials is a field of intense scientific interest. The potentially low production costs and variability in device design such as flexible substrates and color of devices are clear advantages of this emerging technology to standard silicon solar cells. Great progress on device performance could be achieved for organic photovoltaics in the last decade mainly due to the introduction of the bulk heterojunction concept yielding power conversion efficiencies of up to $6 \%$ only recently $[1,2]$. However, superior performance is commonly only reported for devices based on polymers blended with fullerene derivatives. A photovoltaic system based on small molecules appears appealing as materials can be evaporated and materials are mono disperse leading to far enhanced reproducibility of different batches $[3,4]$.

In many recent publications positive effect of thermal or solvent annealing, addition of additives or application of external fields on device performance is observed and mainly attributed to

\footnotetext{
* Corresponding author. Tel.: +49892180 3443; fax: +49 8921803441.

E-mail addresses: Holger.hesse@physik.uni-muenchen.de (H.C. Hesse) Jonas.Weickert@physik.uni-muenchen.de (J. Weickert), m.alhussein@ju.edu.jo (M. Al-Hussein), doessel@mpip-mainz.mpg.de (L. Dössel),

feng@mpip-mainz.mpg.de (X. Feng), muellen@mpip-mainz.mpg.de (K. Müllen), L.Schmidt-Mende@physik.uni-muenchen.de (L. Schmidt-Mende).
}

morphological changes in the photoactive layer [5 7]. However, for a given donor acceptor couple the influence on morphology by such post production treatments is only limited and there is still lack in understanding the relationship between the nano morphology of the active layer and device performance [8]. For the design of novel materials for solar applications more knowl edge to the influence of molecular structure on the assembly of materials and device performance is highly desired.

In this study the assembly of six residue modified donor molecules blended with a commonly used perylene dye as electron acceptor is analyzed. Changes in intermolecular packing and blend morphology by variation of side chain length and structure on the periphery of the donor molecule and their impact on solar cell performance are studied. The materials chosen, hexa peri hexabenzocoronene (HBC) as donor and perylendiimide (PDI) as acceptor, have proven to show potential for efficient photo voltaics [9 12]. HBCs have the potential to self assemble in a columnar fashion resulting in high charge carrier mobility and PDI as electron conducting acceptor is known for its strong absor bance and its organization into supramolecular architectures [12 14].

For the solar cell production an inverted structure with electron collecting $\mathrm{TiO}_{2}$ bottom and $\mathrm{Ag}$ top electrode was chosen. This allows fabrication of devices with highly improved air stability without further device protection such as encapsulation. The measurements of device physics can thus be performed at 
ambient conditions. Additionally, when top contacts are applied by printing techniques, this device design enables vacuum free fabrication, which is an important step towards up scaling and roll to roll processing of devices [15 17]. Details on device fabrication and stability will be shown in a separate publication [18].

\section{Experiments details}

\subsection{Material synthesis}

All materials used in this study are summarized in Scheme 1 and concise abbreviations are given. Synthesis of the hexaphenyl substituted HBCs (Fig. 1, HBC-6, HBC-8 and HBC-12) with different alkyl chain lengths in the periphery was accomplished using a synthesis procedure reported elsewhere [19]. Due to the high sterical demand of the hexadecyl alkyl chain and its limited reactivity a more complex synthetic route was necessary to obtain the HBC-16 (see Supporting information).

To introduce an additional triple bond between the HBC core and the periphery substituents, for compounds $\mathbf{H B C} \equiv \mathbf{1 2}$ and $\mathbf{H B C} \equiv(\mathbf{1 2 , 1 2})$ another synthetic route was used based on a

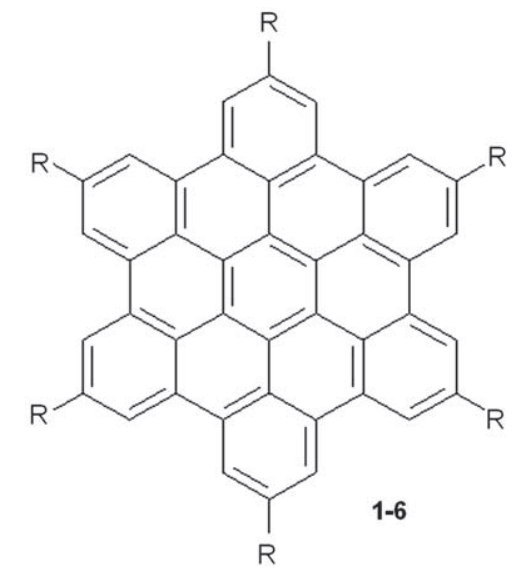
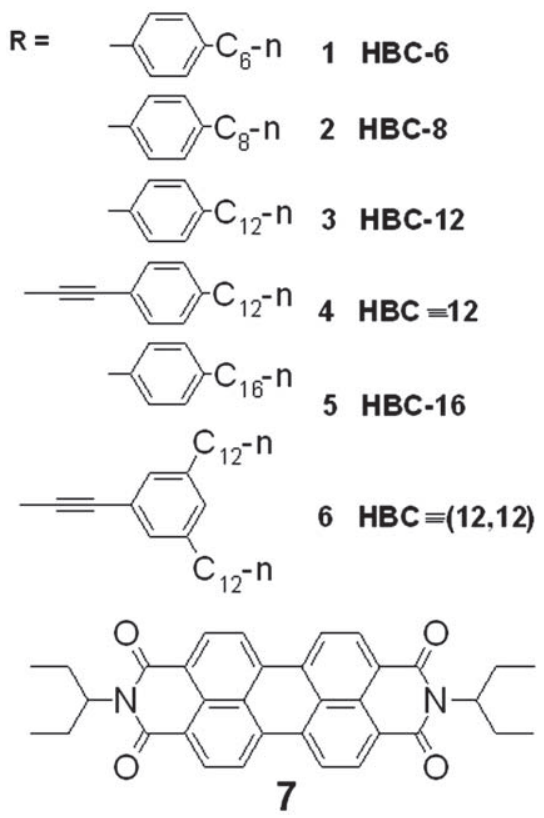

Scheme 1. Chemical structure of organic materials used in solar cells. 1-6: electron acceptor hexa-peri-hexabenzocoronene (HBC) with different residues at the periphery. 7: electron donor perylenediimide (PDI)

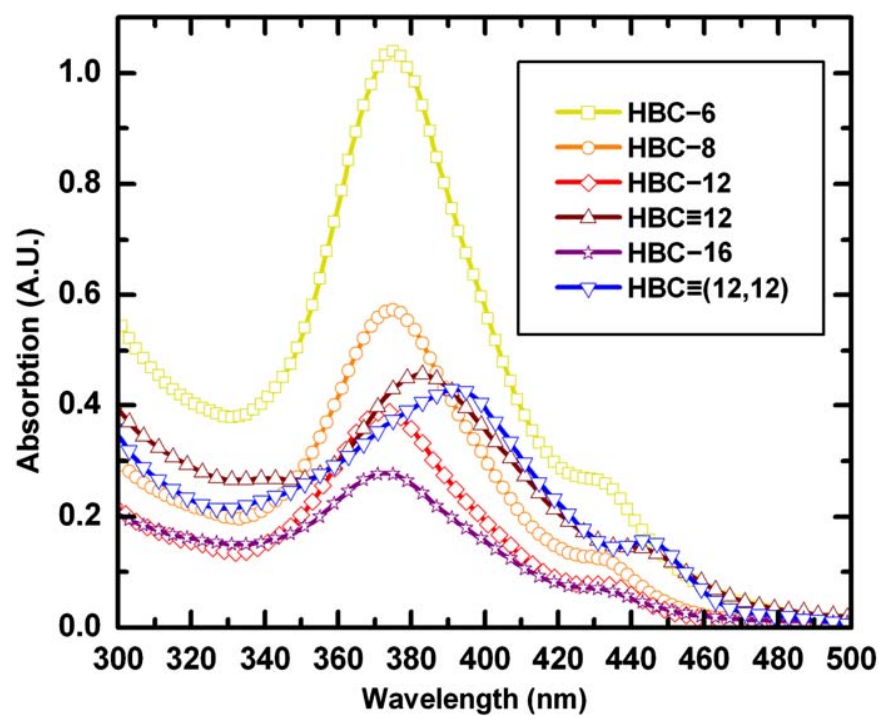

Fig. 1. $H B C$ derivative absorption measured on thin films with comparable thickness of about $100 \mathrm{~nm}$. The HBC peak height is correlated to the number of chromophore molecules. A bathochromic shift is observed for the molecules with a triple bond linker unit.

six fold Sonogashira Hagihara coupling reaction in the last synthetic step.

After synthesis, all compounds were purified by repetitive reprecipitation and silica column chromatography to remove metal ions and impurities that would affect electronic properties and morphology. All synthetic details can be found in the Supporting information.

Perylene tetracarboxdiimide (PDI) was bought from Sensient technologies and used without further purification.

\subsection{Device fabrication}

Devices were fabricated on ITO coated glass substrates. The ITO substrates were cleaned by ultrasonication in acetone and isopropyl alcohol bath followed by a $7 \mathrm{~min} \mathrm{O}_{2}$ plasma (Diener Femto) cleaning. About $150 \mathrm{~nm}$ of $\mathrm{TiO}_{2}$ were deposited onto the cleaned ITO substrates by spray pyrolysis at $450{ }^{\circ} \mathrm{C}$ using a diisopropoxytitanium bis(acetylacetonate) precursor solution. A second plasma cleaning step was carried out for best wetting with the organic material prior to spin coating. HBC and PDI molecules were dissolved in chloroform at a concentration of $15 \mathrm{mg} / \mathrm{ml}$ and mixed at different ratios. Blend solution was stirred for $48 \mathrm{~h}$ and filtered (PTFE, $45 \mu \mathrm{m}$ pore) immediately before use. The organic layers were deposited by spin coating (2000 rpm), resulting in an average film thickness of $\sim 120 \mathrm{~nm}$. PEDOT/PSS was ordered from Sigma Aldrich and applied onto the organic layers by spin coating $(30 \mathrm{~s}, 6000 \mathrm{rpm})$ without subsequent annealing. Top contacts were applied through a shadow mask by sputter coating or evaporation. The cell area was about $0.125 \mathrm{~cm}^{2}$; exact values were determined by microscopy. The devices were annealed for 1 or $14 \mathrm{~h}$ at $120^{\circ} \mathrm{C}$ at ambient atmosphere prior to testing.

\subsection{Absorption and photoluminescence measurements}

Absorption of thin organic layers was measured on Suprasil grade 1 quartz glass with an Agilent Technologies 8453 UV visible spectroscopy system. PL measurements have been conducted on the same samples with a Horiba Yvon Fluorolog PL spectrometer equipped with a $500 \mathrm{~W}$ halogen lamp. 


\subsection{Solar cell characterization}

EQE and IV curves were recorded with a Keithley 2400 Source Meter using a self made LabView based computer program. For calibration a reference silicon diode certified by the Fraunhofer Institute equipped with a KG5 filter was used. For EQE measure ments a $150 \mathrm{~W}$ Xenon lamp was focused onto an Omni150 (LOT Oriel) monochromator $\left(P_{\text {incident }}=0.3 \mathrm{~mW} / \mathrm{cm}^{2}\right.$ at $\left.490 \mathrm{~nm}\right)$. A solar simulator equipped with AM $1.5 \mathrm{~g}$ filters with about $100 \mathrm{~mW} / \mathrm{cm}^{2}$ power was used for measurements.

\subsection{Transient decay experiments}

Photovoltage decay data were recorded under open circuit conditions. White light bias was achieved by a Xenon lamp with a light intensity of about $0.38 \mathrm{~mW} / \mathrm{cm}^{2}$. For both transient measure ments pulsed laser light $(532 \mathrm{~nm}$, pulse frequency $10 \mathrm{~Hz}$, pulse energy about $10 \mu \mathrm{J}$, pulse duration $\sim 4 \mathrm{~ns}$ ) was used to generate small perturbations in the device. The changes in the potential between top and bottom contact of the photovoltaic devices caused by the additional laser pulses were monitored using a digital oscilloscope (Tekscope DPO 7254) with a termination resistance of $1 \mathrm{M} \Omega$.

For the photocurrent decay measurements, blend material of solar cells was excited by pulsed laser irradiation and the time dependent current characteristics recorded via potential drop over the $50 \Omega$ termination of the oscilloscope yielding quasi short circuit conditions. Data were gathered as an average over 100 transient decay events.

\subsection{Surface morphology}

Surface morphology was analysed with a Veeco Dimension 3100 scanning probe microscope. Tap300Al tips with resonance frequency of $300 \mathrm{kHz}$ and force constant of $40 \mathrm{~N} / \mathrm{m}$ were used. Measurements were conducted on the typical annealed devices (glass/ITO/TiO $2 /$ blend/Ag) after removal of the top contacts.

\section{Results and discussion}

\subsection{Material analysis}

The thermotropic properties of the synthesized components were analysed using differential scanning calorimetry (DSC) and $2 \mathrm{D}$ powder $\mathrm{X}$ ray diffraction analysis. All $\mathrm{HBC}$ compounds show a liquid crystalline phase with at least one accessible phase transition (see Supporting information).

Fig. 1 shows absorption spectra of thin films of the analyzed HBC spun from chloroform solution with equal film thickness of about $100 \mathrm{~nm}$. All HBC derivatives show pronounced absorption in the range of $350430 \mathrm{~nm}$. PDI, however, absorbs mostly in the range of $430650 \mathrm{~nm}$ [12]. At constant film thickness strongest absorption of the HBC derivatives is seen for short alkyl side chains. This is attributed to the increased number of chromo phores per volume for small molecular weight HBCs. Both materials with triple bond liker unit, $\mathbf{H B C} \equiv \mathbf{1 2}$ and $\mathbf{H B C} \equiv(\mathbf{1 2}, \mathbf{1 2})$, show bathochromically shifted absorption peaks when compared to the single bond HBCs. Fitting the absorption data to the Urbach model, we could derive a shift in $E_{g}$ of the single bonded to the triple bonded HBC from about 3.1 to about $2.9 \mathrm{eV}$ [20].

\subsection{Solar cell performance}

In order to compare the potential of the different HBC derivatives as donor materials in solar applications, bulk hetero junction solar cells were fabricated, using blends of the HBCs and the acceptor PDI. Solar cells were assembled in an inverted design composed of ITO/TiO $/$ /blend/PEDOT/Ag. With this design strongly improved air stability was achieved compared to the standard ITO/PEDOT/blend/Al layer structure [18,21].

Subsequently devices were annealed at $120^{\circ} \mathrm{C}$ for an extended period of $14 \mathrm{~h}$. Even though this long term annealing treatment results in a slightly decreased absorption for all blend materials (data not shown), drastically improved solar cell performance was recorded showing both improved open circuit voltages and higher short circuit currents. We attribute this to both a favorable realignment of HBC and PDI molecules and the formation of a better contact of the blend with the $\mathrm{Ag}$ electrode as further explained later in connection with AFM measurements.

When illuminated at $1 \mathrm{sun}$ (AM 1.5 global, $100 \mathrm{~mW} / \mathrm{cm}^{2}$ ) all blend cells show photovoltaic behaviour. The effect of donor to acceptor blending ratio on solar cell performance was screened for all derivatives. A mixing ratio of $30: 70 \mathrm{wt} \%$ was found to perform best for $\mathbf{H B C} \equiv(\mathbf{1 2 , 1 2 ) : P D I}$. For short side chain HBCs instead rather equal weight ratio of donor and acceptor yields best results (e.g. HBC-6:PDI with best blend ratio around 50:50 wt\%). To summarize, for best performance the content of HBC core molecules must be decreased significantly when increasing the alkyl chain length or introducing a branched tail. We attribute this to differences in packing and morphology of the different blends. Bulky substituents on the periphery of HBC might hamper intermixing with PDI, as will be further explained later in connection with transient decay measurements and atomic force microscopy. Therefore, higher PDI content is needed to allow for sufficient interfacial area of donor and acceptor yielding maximum performance.

To compare the potential of the different HBCs among themselves, all HBC derivatives were blended with PDI at a constant mixing ratio of 40:60 wt\%. Typical IV Curves at solar illumination (AM $1.5 \mathrm{~g}, 100 \mathrm{~mW} / \mathrm{cm}^{2}$ ) are shown in Fig. 2 and mean values of the solar cell characteristics including standard deviation are summarized in Table 1. Additionally, external quantum efficiency (EQE) measurements have been carried out on all devices. At balanced blend ratio the spectral response mainly follows the absorption of the thin films (exemplary shown for HBC-8:PDI, 40:60 wt\%, inset Fig.2). An EQE of over 27\% was recorded at 390 and $500 \mathrm{~nm}$, which is close to the peak absorption of $\mathrm{HBC}$ and PDI, respectively. For this blend composition the EQE remains above $20 \%$ ranging from 360 to $550 \mathrm{~nm}$.

Best efficiencies of our solar cells are around $\eta=0.24 \%$ upon solar illumination at $100 \mathrm{~mW} / \mathrm{cm}^{2}$ in ambient atmosphere and found to be almost similar for all blends containing HBCs with short side chains (HBC-6, HBC-8 and HBC-12). Upon irradiation with monochromatic light at low intensity about eight times higher efficiencies have been previously reported [12] and were also reproduced with the devices presented here. However, upon increasing light intensity the performance drops significantly. The sub linear increase of currents with illumination intensity is attributed to space charge effects and drastically limits the device performance at solar illumination [22]. The open circuit voltage ranges from 540 to $700 \mathrm{mV}$ and is highest for the HBC derivatives with single bond linked phenyl groups. Within this series of HBCs, the $V_{O C}$ increases from HBC-6 to HBC-12. This can be explained by shielding effects of the alkylic chains decreasing the HOMO level with increasing side chain length as reported elsewhere [23]. Accordingly, the effective bandgap of donor and acceptor $\left(E_{\text {НОмо }}^{D}\right.$ $\left.E_{L U M O}^{A}\right)$ is increased, shifting also the open circuit voltage $\left(V_{O C}\right)$ to higher values [24]. As a different synthetic approach was used and purification appeared more challenging for $\mathbf{H B C}-\mathbf{1 6}$, the low $V_{O C}$ in this blend may be attributed to a higher content of impurities. However, also different molecular arrangement might be favored 


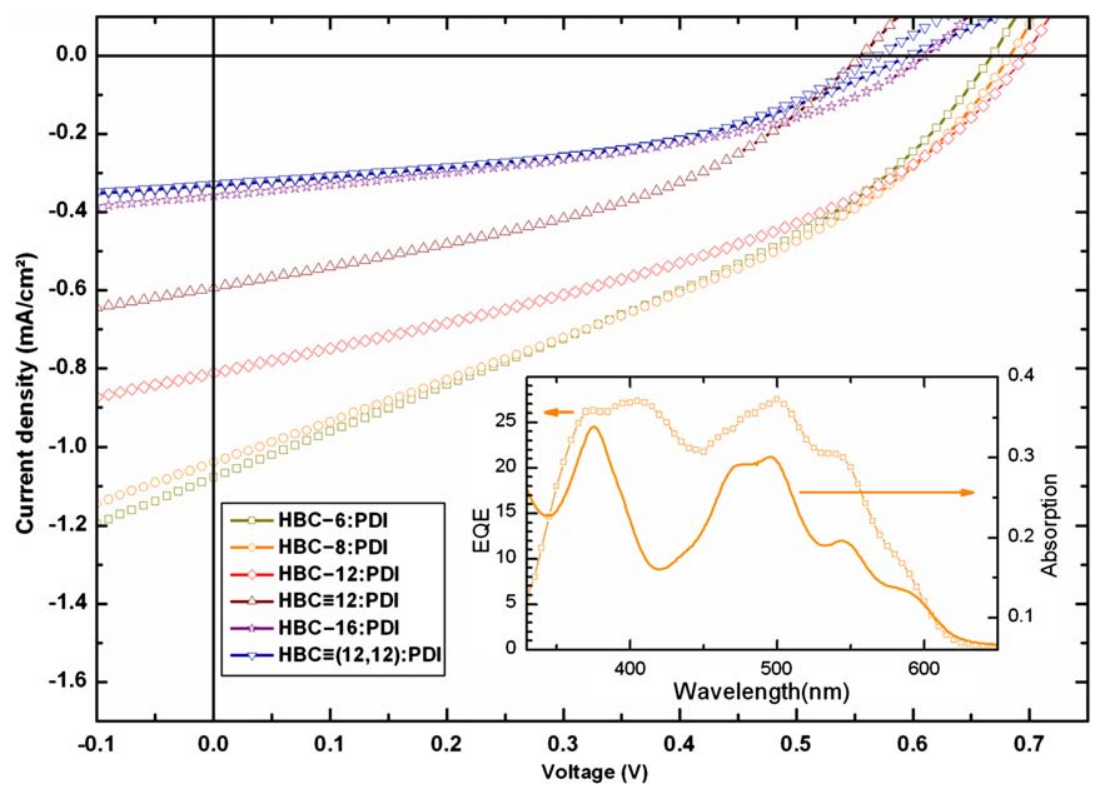

Fig. 2. Typical IV characteristics of different HBC derivatives blended with PDI perylene (40:60 wt\%). Solar cells are measured on air under 1 sun AM1.5 g, $100 \mathrm{~mW} / \mathrm{cm}^{2}$ illumination after thermal annealing. The inset shows absorption data (solid line) and EQE action spectrum (scattered) of a HBC-8:PDI blend at a mixing ratio of (60:40 wt\%).

Table 1

Characteristics of HBC:PDI solar cells. Mean values and standard deviations are given for a set of six cells for each donor-acceptor blend.

\begin{tabular}{|c|c|c|c|c|c|c|}
\hline Cell type & $I_{S C}\left[\mathrm{~mA} / \mathrm{cm}^{2}\right]$ & $V_{O C}[\mathrm{~V}]$ & $\mathrm{FF}[\%]$ & IPCE [\%] & $R_{S}\left[\Omega \mathrm{cm}^{2}\right]$ & $R_{S h}\left[\Omega \mathrm{cm}^{2}\right]$ \\
\hline HBC-6:PDI & $-1.035 \pm 0.042$ & $0.643 \pm 0.023$ & $33.4 \pm 0.2$ & $0.22 \pm 0.02$ & $61 \pm 9$ & $820 \pm 13$ \\
\hline HBC-8:PDI & $-1.065 \pm 0.035$ & $0.665 \pm 0.026$ & $34.3 \pm 0.6$ & $0.24 \pm 0.02$ & $78 \pm 6$ & $906 \pm 28$ \\
\hline HBC-12:PDI & $-0.815 \pm 0.050$ & $0.690 \pm 0.017$ & $39.3 \pm 0.7$ & $0.22 \pm 0.02$ & $57 \pm 12$ & $1608 \pm 127$ \\
\hline $\mathrm{HBC} \equiv 12: \mathrm{PDI}$ & $-0.580 \pm 0.030$ & $0.542 \pm 0.065$ & $38.8 \pm 1.4$ & $0.12 \pm 0.01$ & $138 \pm 69$ & $1997 \pm 215$ \\
\hline HBC-16:PDI & $-0.340 \pm 0.027$ & $0.593 \pm 0.065$ & $39.2 \pm 3.1$ & $0.08 \pm 0.02$ & $76 \pm 35$ & $3729 \pm 324$ \\
\hline $\mathrm{HBC} \equiv(12,12): \mathrm{PDI}$ & $-0.306 \pm 0.037$ & $0.549 \pm 0.041$ & $44.5 \pm 2.9$ & $0.07 \pm 0.02$ & $321 \pm 195$ & $5091 \pm 390$ \\
\hline
\end{tabular}

for this molecule due to the long side chains which could lead to reduced $V_{O C}[25]$.

For both HBCs with a triple bond linker unit about $200 \mathrm{mV}$ lower open circuit voltages are recorded. This can be attributed to altered energy levels for these HBCs. As already mentioned, $E_{g}$ of both $\mathbf{H B C} \equiv \mathbf{1 2}$ and $\mathbf{H B C} \equiv \mathbf{( 1 2 , 1 2 )}$ is shifted by about $0.2 \mathrm{eV}$ to lower values. Electrons can be partially delocalized to the triple bond unit reducing the HOMO level of these molecules. The LUMO level instead is not expected to be influenced by the introduction of the triple bond electrons remain confined to the unmodified HBC core unit [26].

A closer look to the short circuit current $\left(I_{S C}\right)$ indicates differences in the exciton separation and charge carrier collection yield among the different blend materials. Short alkyl chains allow for highest currents and $I_{S C}$ decreases with increasing side chain length of the HBC molecules. Differences in donor acceptor molecular spacing are found to be the main reason as supported by PL measurements and transient photovoltage and photocur rent decay studies.

The fill factor (FF) of the devices ranges from 33.5\% for HBC6:PDI to $42.9 \%$ for $\mathbf{H B C} \equiv(\mathbf{1 2 , 1 2})$ :PDI blends. In a separate experiment $I_{S C}$ of all cells was regulated to an arbitrary but fixed value by adaption of solar illumination intensity. The FF does not considerably differ from that reported in Table 1, showing that FF and values for equivalent resistors strongly depend on material specific properties and are not governed by contact limitations.

Both shunt and series resistances for an equivalent diode model are calculated from the IV curves (Table 1 ). The shunt resistance $\left(R_{S h}\right)$ ranges from $10^{3}$ to $10^{4} \Omega \mathrm{cm}^{2}$, which is compar able to values reported in solar cells based on other organic materials [27]. However, especially for the devices with short alkyl chain HBCs increasing the shunt resistance is of key importance for further device improvement. Instead, high series resistance $\left(R_{S}\right)$ of about $60320 \Omega \mathrm{cm}^{2}$ was recorded for the cells which is at least one order of magnitude larger than common values reported for morphologically optimized polymer fullerene solar cells $[1,27]$. High $R_{S}$ values cause major limitations to both the FF and $I_{S C}$ of our devices, especially for cells containing $\mathbf{H B C} \equiv \mathbf{1 2}$ and particularly $\mathbf{H B C} \equiv(\mathbf{1 2 , 1 2})$. Higher mobility in the blend material is needed for improve short circuit currents and $\mathrm{FF}$ as will be further motivated later in connection with transient decay measurements. In highly aligned material record mobility of up to $1 \mathrm{~cm}^{2} \mathrm{~V}^{1} \mathrm{~s}^{1}$ has been achieved in HBC and PDI thin films along the $\pi \pi$ axis $[13,28,29]$. However, in solar cells homeotropic alignment and crystal growth are necessary to yield a high mobility and allow for efficient percolation pathways. This favorable alignment and morphology is most likely not achieved in the devices studied. As already mentioned significant space charge effects are observed under normal working conditions indicating only insufficient mobility in at least one of the organic materials used. Furthermore, the IV curve analysis also indicates that optimum alignment is not achieved in our devices yet: in fact, it appears that lateral charge carrier hopping between adjacent HBC cores is necessary for current generation. This hopping process should be most probable for the hexagonally and closely packed HBCs with short side chains and might be hindered 
for $\mathbf{H B C} \equiv \mathbf{1 2}$ and $\mathbf{H B C} \equiv(\mathbf{1 2 , 1 2})$ with their more space demanding cubic and herringbone packing (see Table S1 in Supporting information) giving rise to increased series resistance in these devices.

\subsection{Photoluminescence analysis}

Photoluminescence (PL) measurements have been carried out on thin films of both pure PDI and HBC derivative films as well as on the blended compounds and their quenching analyzed. For the blends, measurements were conducted on the fully assembled solar cell devices after annealing in reflection geometry. Thus direct insight to the spectral properties of the materials used in the solar cells presented in Table 1 was gained. Excitation was set to 375 and $490 \mathrm{~nm}$, where prominent absorption takes place in the HBC and PDI molecules, respectively. PL signals were spectrally resolved as exemplary shown for excitation at $375 \mathrm{~nm}$ in Fig. 3.

When blend material is excited at $375 \mathrm{~nm}$ broad features are visible at 525 and $610 \mathrm{~nm}$ matching well the emission of excited pure HBC and PDI films, respectively. Both HBC and predomi nantly PDI emission are visible for all blends indicating efficient energy transfer from the HBC to PDI. Interestingly, the feature at around $750 \mathrm{~nm}$ is not visible for thin films prepared of either pure HBC or PDI. We attribute this signal to a photo induced charge transfer state (CTS) [30]. In our experiments the feature could only be resolved for blends containing the HBCs with single bond linker unit. For both triple bond materials the altered energy gap is likely to induce a red shift for the CTS, which could not be resolved with our experimental setup [30].

Upon excitation of PDI $(490 \mathrm{~nm})$ all emission curves of the blend materials look very similar in shape varying only in the overall height with stronger quenching for short alkyl chains (data not shown). Typical PDI related emission features are visible but no signal from HBC emission could be recognized. The CTS signal at around $725 \mathrm{~nm}$ is still present for this excitation but decreased in intensity. Furthermore, photoluminescence excitation mea surements have shown that the spectral contribution to the CTS mainly follows the absorption with higher yield upon $\mathrm{HBC}$ excitations.

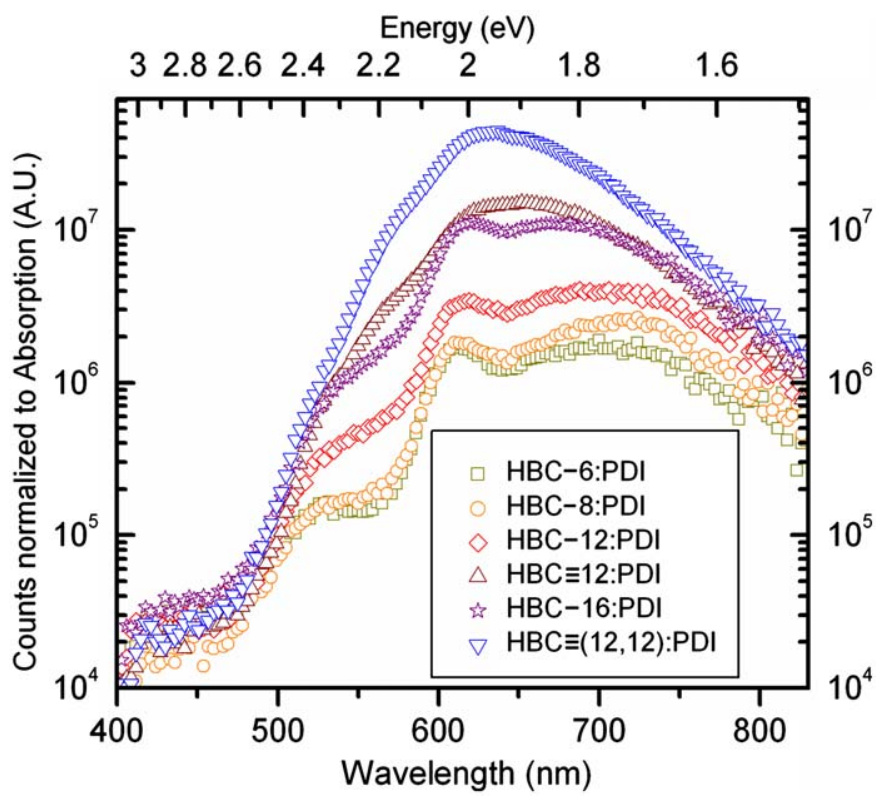

Fig. 3. Emission photoluminescence spectrum of devices comprised of different blend materials. Samples were excited at $375 \mathrm{~nm}$ in reflection geometry. Detector counts are normalized to the sample absorption.
Results of the PL measurements can be explained when looking at the energy levels of donor and acceptor molecules. As known from literature, the HOMO levels of HBC and PDI are very similar at around $5.3 \mathrm{eV}[12]$. When blend material is excited at $375 \mathrm{~nm}$ the offset in LUMO levels of about $0.65 \mathrm{eV}$ leads to both separation of excitons at the interface and energy transfer to the PDI. Upon excitation at $490 \mathrm{~nm}$ instead most emission occurs directly from the PDI. Despite the low driving force due to the small offset in HOMO levels a significant number of excitons is separated at the interface resulting in a spectral contribution of the EQE at wavelengths above $450 \mathrm{~nm}$. In addition, some radiative loss of CTS excitons can be detected upon PDI excitation.

The overall radiative loss in the different blends can be derived from the absorption normalized area under the curve of the PL signals (Fig. 3). At both excitation wavelengths probed, best quenching is observed for HBCs with short alkyl chains, which is attributed to morphological differences in the thin films. The distance between PDI molecules and the HBC cores is likely to depend on the residue variations of the $\mathrm{HBC}$ molecules similar to the inter core distances measured for pure HBCs (Table S1 in Supporting information). Furthermore, better intermixing of donor and acceptor for short side chain HBCs is supported by AFM data. Intimate contact of HBC cores and the PDI is needed to efficiently separate excitons also allowing for higher photocur rents (as reported in Table 1 and Fig. 2). For long and especially swallow tailed alkyl side chains, relaxation of excited molecules by charge separation becomes less probable. Instead, charge carriers recombine and emit their energy in terms of photo luminescence giving rise to lower currents as observed in the IV curves. Generally the triple bond linker unit present in $\mathbf{H B C} \equiv \mathbf{1 2}$ and $\mathbf{H B C} \equiv(\mathbf{1 2 , 1 2})$ appears to widen the radiative loss channel of the blends. The overall higher sterical demand of these HBCs is likely to be the reason: The phenyl group is located further away from the $\mathrm{HBC}$ core unit resulting in a less compact packing in $\mathbf{H B C} \equiv \mathbf{1 2}$ (cubic lattice) and $\mathbf{H B C} \equiv \mathbf{( 1 2 , 1 2 )}$ (tilted herringbone structure) (see Table S1 in Supporting information).

\subsection{Transient decay measurements}

Two types of transient decay measurements were performed on the solar cell devices in order to gain insight to charge carrier recombination and extraction.

Fig. 4 shows a typical photovoltage decay (PVD) transient monitored with a digital high resolution oscilloscope. In addition to the potential generated by white light illumination, supplementary charge carriers are created by short laser pulses $(532 \mathrm{~nm})$ raising also the open circuit potential of the cell temporarily. The voltage drops to the former level due to recombination of the additional charge carriers. The decay process can be described by a bi exponential function,

$V_{\text {Bias }}=A_{\text {fast }} e^{-k_{\text {fast }} t}+A_{\text {slow }} e^{-k_{\text {slow }} t}$

with decay rates $k_{\text {fast }}$ and $k_{\text {slow }}$ differing by about one order of magnitude and respective constants $A_{\text {fast }}$ and $A_{\text {slow }}$. Investigations at different laser pulse intensities have shown that $k_{\text {fast }}$ strongly depends on the perturbing light intensity and does not reveal blend specific properties [31]. The slow decay rate instead gives insight to the material specific recombination of the solar cells: for cells under working conditions an $e^{1}$ lifetime of charges can be estimated from the decay rate as

$\tau_{1 / e}=k_{\text {slow }}{ }^{-1}$

Annealed devices with blends of HBC:PDI (40:60 wt\%) were analyzed and transients show lifetimes in the range of a few tens of microseconds, which is comparable to common values found 


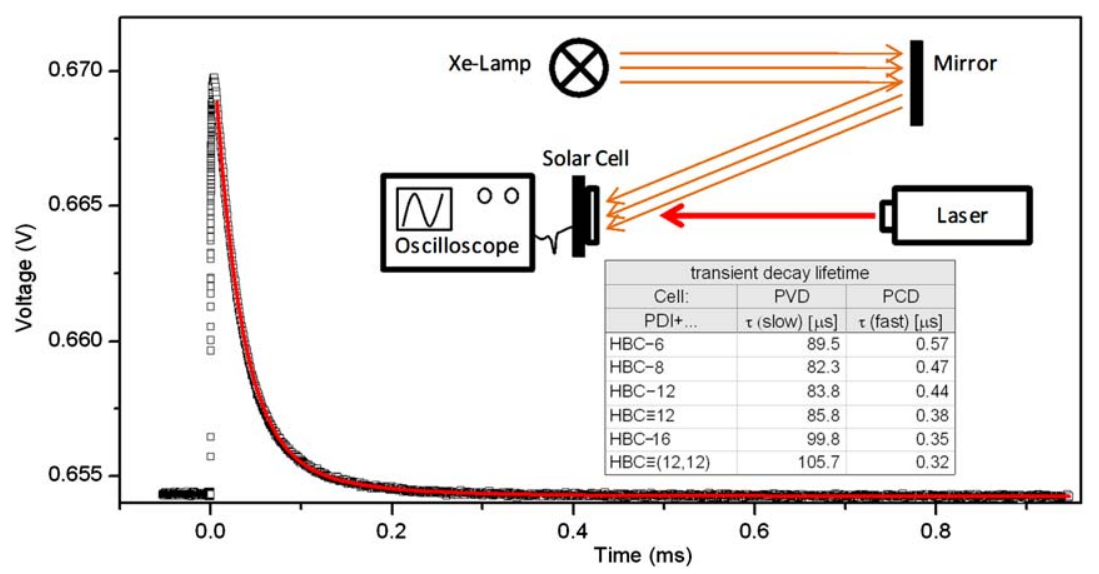

Fig. 4. Photovoltage and photocurrent decay experiments. Inset cartoon depicts the setup geometry. Exemplary curve shows photovoltage decay of a HBC-6:PDI cell (scattered) and the according bi-exponential fit (red line). The inset table summarizes decay rates $k_{\text {slow }}$ and $k_{\text {fast }}$ for photovoltage (PVD) and photocurrent (PCD) decay, respectively. (For interpretation of the references to color in this figure legend, the reader is referred to the web version of this article.)

for other organic photovoltaic systems (inset table, Fig. 4) [32]. Charge recombination occurs faster in blends containing short side chain HBCs, which further explains the differences observed in $R_{S h}$ and FF for reported in Table 1: both FF and $R_{S h}$ are found to decrease gradually from long to short residues at the periphery of the HBCs, implying more pronounced recombination and in creased leakage currents. Even though charge separation is not favored in the $\mathbf{H B C} \equiv(\mathbf{1 2 , 1 2})$ containing blends manifested by the low $I_{S C}$ and high PL loss, once excitons are separated in this blend, generated charges can efficiently be extracted and recombination is only a minor loss mechanism. For the cells containing HBC-6 instead, considerably more free carriers are produced yielding a higher short circuit current. However, devices suffer strong recombination resulting in the low FF of these devices.

To further analyze the charge carrier generation and extrac tion, photocurrent decay measurements (PCD) were also carried out on the solar cell devices. Contrary to the PVD experiments, measurements were conducted under short circuit conditions with no white light bias. Transients recorded are also subject to a bi exponential decay and can be fitted with Eq. (1). For PCD measurements, slow decay rates are strongly influenced by trap states and only the short lived state incorporates blend specific information [33]. An $e^{1}$ lifetime is extracted from the fast decay rates (Fig.4, inset table). Reported charge extraction rates are two orders of magnitude faster than respective recombination rates determined by the PVD measurements a prerequisite for efficient charge carrier extraction in the solar cell devices: separated charges will rather exit the device via the external $\mathrm{TiO}_{2}$ and $\mathrm{Ag}$ contacts than become annihilated within the active material.

All materials show a peak current response to the laser pulse of similar magnitude. However, the decay from this peak value occurs faster in the blends with long or branched side chain HBCs. The total amount of charge $(Q)$ generated by one laser pulse on a specific cell is equal to the area under the decay curve ( $I$ vs. $t$ ). $Q$ increases from $\mathbf{H B C} \equiv(\mathbf{1 2 , 1 2})$ to $\mathbf{H B C}-\mathbf{6}$, which is consistent with the differences in short circuit currents of the $I V$ measurements presented (Fig. 2). Upon laser irradiation at $532 \mathrm{~nm}$, only PDI molecules become excited which can either contribute to charge carrier generation, or unwanted relaxation occurs. In the blends containing long residue HBCs prominent non radiative and radiative relaxation of the excited PDI molecules are indicated by fast decay rates. For HBCs with short chain residues instead, intimate contact of the HBC core to the excited PDI is provided, recombination is suppressed and charges are generated over a longer period of time. This results in the slow decay rates recorded and is also in accordance with the PL signals quantifying the radiative recombination loss channel. It appears that the capability to extract more charges from the excited PDI outweighs the faster recombination for blends containing short alkyl chain HBCs as shown with PVD measurements and explains the significantly higher short circuit currents of these materials [34].

The PCD decay rates reported for the HBC:PDI devices are comparable to those found in literature for high efficient polymer fullerene devices[34]. However, peak current offset after pertur bation and thus the overall extracted charge is found significantly lower for the small molecule devices presented here. Optimiza tion of alignment in the discotic blend material will lead to improved mobility and allow for faster charge carrier extraction making these small molecule solar cells competitive with morphology optimized polymer fullerene devices.

\subsection{Surface morphology analysis}

Tapping mode atomic force microscopy (AFM) has been carried out on the different organic blend layers studied. Images were taken on the identical samples which yielded the above shown $I V$ characteristics after careful removal of the Ag top contact. Thus surface morphology of the different blends could be analyzed exactly where the material was previously confined between bottom and top electrode.

In open films, PDI forms long crystalline grains. Pure HBC films exhibit rather smooth and almost featureless surface structures instead [12]. Throughout the annealing treatment at $120^{\circ} \mathrm{C}$ PDI is in isotropic phase and also side chains of the liquid crystalline HBCs are mobile (Table S1 in Supporting information). As the blend material was kept in confinement sandwiched between the $\mathrm{TiO}_{2}$ and $\mathrm{Ag}$ electrode the formation of PDI needles could be effectively suppressed yielding RMS roughness values of only $47 \mathrm{~nm}$ for the different HBC:PDI blends studied. The confined annealing treatment leads to an improved morphology and is favorable for device performance. In non confined geometry instead, PDI needles are likely to penetrate through the entire active layer establishing direct contact between both electrodes $[12,35]$. Increase leakage currents and reduced shunt resistance are observed.

Furthermore, during thermal annealing also the nano mor phology of the blend material changes: realignment of the discotic molecules leads to increased ordering [36,37]. An altered 

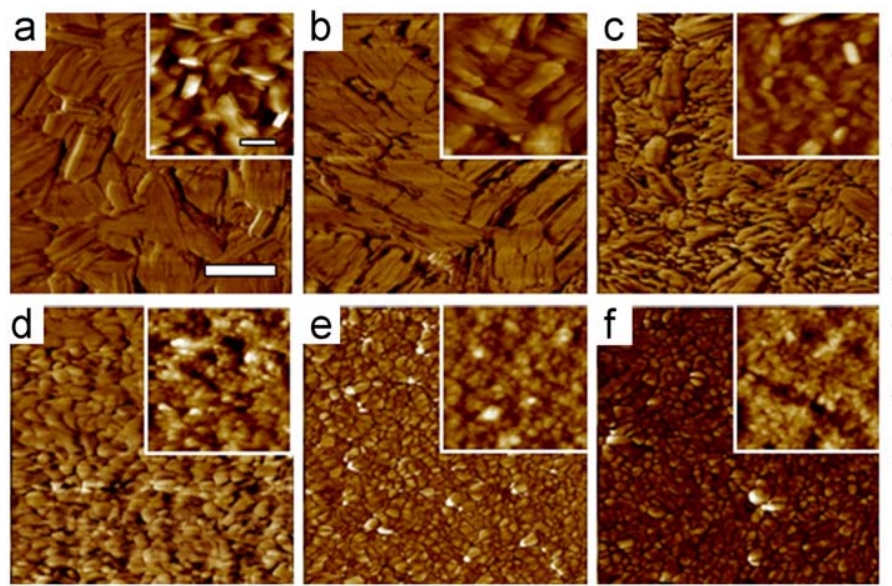

\begin{tabular}{|l|l|}
\hline Sample & Cell type \\
\hline a) & HBC-6:PDI \\
\hline b) & HBC-8:PDI \\
\hline c) & HBC-12:PDI \\
\hline d) & HBC $\equiv 12: P D I$ \\
\hline e) & HBC-16:PDI \\
\hline f) & $\begin{array}{l}\text { HBC }:(12,12) \\
\text { PDI }\end{array}$ \\
\hline & 25 \\
\hline & $50 \mathrm{~nm}$ \\
\hline 0 & 25 \\
\hline
\end{tabular}

Fig. 5. AFM tapping mode scans of annealed $\left(14 \mathrm{~h}, 120^{\circ} \mathrm{C}\right) \mathrm{HBC}$ :PDI $40: 60 \mathrm{wt} \%$ samples. Scale bar is $500 \mathrm{~nm}$ for both phase (large image) and height scans (insets). Images were taken after top contact removal and give insight to the blend morphology present in solar cell devices. Crystalline structures are observed mainly for short alkyl side chains at the periphery of $\mathrm{HBC}$.

morphology showing larger grains can be visualized with AFM scans. The observed increase of device performance as already reported along with the $I V$ curves is attributed to better percolation pathways. However, when annealed for over $24 \mathrm{~h}$ decrease of short circuit currents was observed. We attribute this to well advanced de mixing and consequently reduced interfacial area of HBC with PDI [38].

After annealing in confined geometry the blend materials studied also show differences in surface topology as summarized in Fig. 5. Flake like structures are visible for all derivatives, whereas the grain size decreases from about $500 \mathrm{~nm}$ (for HBC-6 and $\mathbf{H B C}-8$ ) to $100 \mathrm{~nm}$ (for $\mathbf{H B C}-16$ and $\mathbf{H B C} \equiv(\mathbf{1 2 , 1 2})$ ) in diameter. For longer side chain HBCs, the domains are interrupted by less ordered areas. This may also lead to a lower charge carrier mobility and could thus cause the lower currents observed for these materials. In contrast, the larger grains for the short side chain HBCs rather consist of better aligned material. Initial $\mathrm{X}$ ray experiments show, that most likely a mixed phase of HBC and PDI is present in this grains. The increased crystallite size allows for better percolation pathways towards the contacts. Intermixing of HBC and PDI is necessary for efficient exciton separation upon illumination. These findings are consistent with both PL data and the PCD measurements indicating that the charge carrier generation yield is higher when incorporating short side chain molecules.

The surface morphology of the active material indicates differences in cristallinity and quality of crystal growth. These parameters play an important role for the solar cell performance consisting of discotic molecules. For efficient devices large and ordered domains incorporating intermixed donor and acceptor material are necessary.

\section{Conclusion}

We have studied the influence of alkyl side chain modifications of discotic liquid crystalline donor molecules for solar cell applications. Short alkyl tails allow for a more intimate contact of the HBC cores to a PDI acceptor molecule. PCD and PL measurements indicate that radiative and non radiative loss mechanisms of excited molecules can be efficiently suppressed when using $\mathrm{HBC}$ with shorter side chains, where consequently best exciton separation and highest power conversion efficiencies are found. AFM measurements explain the significance of annealing in confinement and reveal differences in cristallinity of the different blends investigated. The findings also support the formation of an intermixed donor acceptor phase for short chain HBCs. Thus large interfacial area between donor and acceptor and at the same time high mobility in the respective materials can be achieved. With this donor acceptor couple external quantum efficiency of over $27 \%$ was achieved.

Additionally, our studies reveal pathways to further increase the device performance. First of all, a more adequate matching of HOMO and LUMO levels is necessary to increase charge transfer and suppress energy transfer and radiative recombination as observed in our devices. Currently, charge carrier generation is limited by high radiative loss as indicated by PL data. Furthermore PL measurements point out that the phenyl groups should remain in close contact to the $\mathrm{HBC}$ core since a new radiative loss channel is introduced by a larger spacing due to a triple bond linker unit. Also higher mobility within the blend material is needed to allow for faster charge carrier extraction and accord ingly lower recombination as motivated by our findings based on PVD data.

Most important will be a good hometropic alignment of the $\mathrm{HBC}$ molecules in the active film. Face on aligned molecules will facilitate faster charge transport towards the electrodes leading to improved charge extraction [39]. In conjunction with molecules having long alkyl chains and consequently a lower charge carrier recombination as supported by our transient decay measure ments improved external quantum efficiency and higher fill factor are expected. Thus, our results suggest that the full potential of discotic molecules can only be accessed when material is fully homeotropically aligned and recombination is suppressed by an appropriate intermolecular distance.

\section{Acknowledgements}

We would like to thank the German research foundation (DFG) for funding in the program "SPP1355: elementary processes of organic photovoltaics" as well as funding in the Cluster of Excellence "Nanosystems Initiative Munich (NIM)". We would like to thank the groups of Prof. Feldmann and Prof. Kotthaus giving us access to their equipment (PL measurements, metal evaporator, glove box and AFM, respectively).

We thank Enrico DaComo and Markus Hallermann for their fruitful discussions and support for PL measurements. 


\section{Appendix A. Supporting information}

Supplementary data associated with this article can be found in the online version at doi:10.1016/j.solmat.2009.11.024.

\section{References}

[1] G. Li, V. Shrotriya, J.S. Huang, Y. Yao, T. Moriarty, K. Emery, Y. Yang, Highefficiency solution processable polymer photovoltaic cells by self-organization of polymer blends, Nature Materials 4 (2005) 864-868.

[2] S.H. Park, A. Roy, S. Beaupre, S. Cho, N. Coates, J.S. Moon, D. Moses, M. Leclerc, K. Lee, A.J. Heeger, Bulk heterojunction solar cells with internal quantum efficiency approaching 100\%, Nature Photonics 3 (2009) 297-302.

[3] R. Schueppel, K. Schmidt, C. Uhrich, K. Schulze, D. Wynands, J.L. Bredas, E. Brier, E. Reinold, H.B. Bu, P. Baeuerle, B. Maennig, M. Pfeiffer, K. Leo, Optimizing organic photovoltaics using tailored heterojunctions: a photoinduced absorption study of oligothiophenes with low band gaps, Physical Review B 77 (2008) 085311-1-085311-14.

[4] B. Rand, J. Genoe, P. Heremans, J. Poortmans, Solar cells utilizing small molecular weight organic semiconductor, Progress in Photovoltaics 15 (2007) 659-676.

[5] G. Li, V. Shrotriya, Y. Yao, Y. Yang, Investigation of annealing effects and film thickness dependence of polymer solar cells based on poly(3-hexylthiophene), Journal of Applied Physics 98 (2005) 43704-43705.

[6] S. Miller, G. Fanchini, Y.-Y. Lin, C. Li, C.-W. Chen, W.-F. Su, M. Chhowalla, Investigation of nanoscale morphological changes in organic photovoltaics during solvent vapor annealing, Journal of Materials Chemistry 18 (2008) 306-312.

[7] F. Padinger, R.S. Rittberger, N.S. Sariciftci, Effects of postproduction treatment on plastic solar cells, Advanced Functional Materials 13 (2003) 85-88.

[8] X. Yang, J. Loos, Toward high-performance polymer solar cells: the importance of morphology control, Macromolecules 40 (2007) 1353-1362.

[9] W.S. Shin, H.H. Jeong, M.K. Kim, S.H. Jin, M.R. Kim, J.K. Lee, J.W. Lee, Y.S. Gal, Effects of functional groups at perylene diimide derivatives on organic photovoltaic device application, Journal of Materials Chemistry 16 (2006) 384-390.

[10] Z.J. Chen, M.G. Debije, T. Debaerdemaeker, P. Osswald, F. Wurthner, Tetrachloro-substituted perylene bisimide dyes as promising n-type organic semiconductors: studies on structural, electrochemical and charge transport properties, Chemphyschem 5 (2004) 137-140.

[11] P.E. Keivanidis, I.A. Howard, R.H. Friend, Intermolecular interactions of perylene diimides in photovoltaic blends of fluorene copolymers: disorder effects on photophysical properties, film morphology and device efficiency, Advanced Functional Materials 18 (2008) 3189-3202.

[12] L. Schmidt-Mende, A. Fechtenkotter, K. Mullen, E. Moons, R.H. Friend, J.D. MacKenzie, Self-organized discotic liquid crystals for high-efficiency organic photovoltaics, Science 293 (2001) 1119-1122.

[13] S. Sergeyev, W. Pisula, Y.H. Geerts, Discotic liquid crystals: a new generation of organic semiconductors, Chemical Society Reviews 36 (2007) 1902-1929.

[14] F. Wurthner, Perylene bisimide dyes as versatile building blocks for functional supramolecular architectures, Chemical Communications 14 (2004) 1564-1579.

[15] F.C. Krebs, All solution roll-to-roll processed polymer solar cells free from indium-tin-oxide and vacuum coating steps, Organic Electronics 10 (2009) 761-768.

[16] F.C. Krebs, M. Jørgensen, K. Norrman, O. Hagemann, J. Alstrup, T.D. Nielsen, J. Fyenbo, K. Larsen, J. Kristensen, A complete process for production of flexible large area polymer solar cells entirely using screen printing-first public demonstration, Solar Energy Materials and Solar Cells 93 (2009) 422-441.

[17] F.C. Krebs, S. Gevorgyan, J. Alstrup, A roll-to-roll process to flexible polymer solar cells: model studies, manufacture and operational stability studies, Journal of Materials Chemistry 19 (2009) 5442-5451.

[18] H.C. Hesse, J. Weickert, H. Sun, L. Schmidt-Mende, Air stability and degradation of inverted organic solar cells, unpublished work.
[19] A. Fechtenkoetter, K. Saalwaechter, M. Harbison, K. Muellen, H. Spiess, Highly ordered columnar structures from hexa-peri-hexabenzocoronenessynthesis, X-ray diffraction, and solid-state heteronuclear multiple-quantum NMR investigations, Angewandte Chemie International Edition 38 (1999) 3039-3042.

[20] D.C. Olson, S.E. Shaheen, M.S. White, W.J. Mitchell, M.F.A.M. van Hest, R.T. Collins, D.S. Ginley, Band-offset engineering for enhanced open-circuit voltage in polymer-oxide hybrid solar cells, Advanced Functional Materials 17 (2007) 264-269.

[21] C.S. Kim, S.S. Lee, E.D. Gomez, J.B. Kim, Y.L. Loo, Transient photovoltaic behavior of air-stable, inverted organic solar cells with solution-processed electron transport layer, Applied Physics Letters 94 (2009) 113302.

[22] V. Mihailetchi, J. Wildeman, P. Blom, Space-charge limited photocurrent, Physical Review Letters 94 (2005) 126602.

[23] J. Li, M. Kastler, W. Pisula, J.W.F. R.obertson, D. Wasserfallen, A.C. Grimsdale, J. $\mathrm{Wu}, \mathrm{K}$. Mullen, Organic bulk-heterojunction photovoltaics based on alkyl substituted discotics, Advanced Functional Materials 17 (2007) 2528-2533.

[24] M.C. Scharber, D. Wuhlbacher, M. Koppe, P. Denk, C. Waldauf, A.J. Heeger, C.L. Brabec, Design rules for donors in bulk-heterojunction solar cells-towards $10 \%$ energy-conversion efficiency, Advanced Materials 18 (2006) 789-794.

[25] C. Videlot, D. Fichou, Influence of molecular orientation on the photovoltaic properties of octithiophene, Synthetic Metals 102 (1999) 885-888.

[26] I. Gutman, Z. Tomovic, K. Müllen, J.P. Rabe, On the distribution of [pi]electrons in large polycyclic aromatic hydrocarbons, Chemical Physics Letters 397 (2004) 412-416.

[27] M.S. Kim, B.G. Kim, J. Kim, Effective variables to control the fill factor of organic photovoltaic cells, Acs Applied Materials \& Interfaces 1 (2009) $1264-1269$

[28] A.M. van de Craats, J.M. Warman, A. Fechtenkotter, J.D. Brand, M.A. Harbison, Record charge carrier mobility in a room-temperature discotic liquidcrystalline derivative of hexabenzocoronene, K Mullen, Advanced Materials 11 (1999) 1469-1472.

[29] W. Pisula, A. Menon, M. Stepputat, I. Lieberwirth, U. Kolb, A. Tracz, H. Sirringhaus, T. Pakula, K. Mullen, A zone-casting technique for device fabrication of field-effect transistors based on discotic hexa-perihexabenzoeoronene, Advanced Materials 17 (2005) 684-689.

[30] D. Veldman, S.C.J. M.eskers, R.A.J. J.anssen, The energy of charge-transfer states in electron donor-acceptor blends: insight into the energy losses in organic solar cells, Advanced Functional Materials 19 (2009) 1939-1948.

[31] N.W. Duffy, L.M. Peter, K.G.U. W.ijayantha, Characterisation of electron transport and back reaction in dye-sensitised nanocrystalline solar cells by small amplitude laser pulse excitation, Electrochemistry Communications 2 (2000) 262-266.

[32] L.J.A. K.oster, V.D. Mihailetchi, P.W.M. B.lom, Bimolecular recombination in polymer/fullerene bulk heterojunction solar cells, Applied Physics Letters 88 (2006) 052104

[33] M.A. Fox, H.L. Pan, W.E. Jones, D. Melamed, Spectroscopy and time-resolved photocurrent response in ordered porphyrin thin-films, Journal of Physical Chemistry 99 (1995) 11523-11530.

[34] C.G. Shuttle, A. Maurano, R. Hamilton, B. O’Regan, J.C. de Mello, J.R. Durrant, Charge extraction analysis of charge carrier densities in a polythiophene/ fullerene solar cell: analysis of the origin of the device dark current, Applied Physics Letters 93 (2008) 183501-1-183501-3.

[35] J.P. Schmidtke, R.H. Friend, M. Kastler, K. Mullen, Control of morphology in efficient photovoltaic diodes from discotic liquid crystals, Journal of Chemical Physics 124 (2006) 174704-1-174704-6.

[36] W. Pisula, M. Kastler, B. El Hamaoui, M.C. Garcia-Gutierrez, R.J. Davies, C. Riekel, K. Mullen, Dendritic morphology in homeotropically aligned discotic films, Chemphyschem 8 (2007) 1025-1028.

[37] X. Yang, J. Loos, S.C. Veenstra, W.J.H. V.erhees, M.M. Wienk, J.M. Kroon, M.A.J. M.ichels, R.A.J. J.anssen, Nanoscale morphology of high-performance polymer solar cells, Nano Letters 5 (2005) 579-583.

[38] S. Bertho, I. Haeldermans, A. Swinnen, W. Moons, T. Martens, L. Lutsen, D. Vanderzande, J. Manca, A. Senes, A. Bonfiglio, Influence of thermal ageing on the stability of polymer bulk heterojunction solar cells, Solar Energy Materials and Solar Cells 91 (2007) 385-389.

[39] C. Videlot, D. Fichou, Influence of molecular orientation on the photovoltaic properties of octithiophene, Synthetic Metals 102 (1999) 885-888. 